\title{
IR780-loaded folate-targeted nanoparticles for near-infrared fluorescence image-guided surgery and photothermal therapy in ovarian cancer
}

\author{
Jiao Song ${ }^{1,2}$ \\ Ni Zhang ${ }^{1,2}$ \\ Liang Zhang ${ }^{2,3}$ \\ Hengjing $\mathrm{Yi}^{2}$ \\ Yi Liu ${ }^{1,2}$ \\ Yanxi $\mathrm{Li}^{1}$ \\ Xuelin $\mathrm{Li}^{2,4}$ \\ Meng $\mathrm{Wu}^{2,5}$ \\ Lan $\mathrm{Hao}^{2,3}$ \\ Zhu Yang' \\ Zhigang Wang ${ }^{2,3}$ \\ 'Gynaecology and Obstetrics \\ Department, The Second Affiliated \\ Hospital of Chongqing Medical University, \\ Chongqing 4000 10, People's Republic of \\ China; ${ }^{2}$ Chongqing Key Laboratory of \\ Ultrasound Molecular Imaging, \\ Chongqing Medical University, Chongqing \\ 4000 10, People's Republic of China; \\ ${ }^{3}$ Ultrasound Department, The Second \\ Affiliated Hospital of Chongqing Medical \\ University, Chongqing 4000 I0, People's \\ Republic of China; ${ }^{4}$ Geriatric \\ Department, Chongqing General \\ Hospital, Chongqing 400014, People's \\ Republic of China; ${ }^{5}$ Ultrasound \\ Department, Zhongnan Hospital of \\ Wuhan University, Hubei 43007I, \\ People's Republic of China
}

Correspondence: Zhu Yang

Gynaecology and Obstetrics Department,

The Second Affiliated Hospital of

Chongqing Medical University, Chongqing

400010 , People's Republic of China;

Tel +86236848 6646

Fax +862368485826

Email yangzhu@hospital.cqmu.edu.cn

Zhigang Wang

Ultrasound Department, The Second

Affiliated Hospital of Chongqing Medical

University, Chongqing, 4000 10, People's

Republic of China

Tel +862363809074

Fax +862363809074

Email wzg62942443@163.com
This article was published in the following Dove Press journal:

International Journal of Nanomedicine

Background and purpose: Surgery is regarded as the gold standard for patients with advanced ovarian cancer. However, complete surgical removal of tumors remains extremely challenging; fewer than $40 \%$ of patients are cured. Here, we developed a new modality of theranostics for ovarian cancer based on a near-infrared light-triggered nanoparticle.

Methods: Nanoparticles loading IR780 iodide on base of folate modified liposomes were prepared and used for theranostics of ovarian cancer. Tumor targeting of FA-IR780-NP was evaluated in vitro and in an ovarian xenograft tumor model. A fluorescence stereomicroscope was applied to evaluate the tumor recognition of FA-IR780-NP during surgery. FA-IR780-NP mediated photothermal therapy effect was compared with other treatments in vivo.

Results: FA-IR780-NP was demonstrated to specifically accumulate in tumors. IR780 iodide selectively accumulated in tumors; the enhanced permeability and retention effect of the nanoparticles and the active targeting of folate contributed to the excellent tumor targeting of FA-IR780-NP. With the aid of tumor targeting, FA-IR780-NP could be used as an indicator for the real-time delineation of tumor margins during surgery. Furthermore, photothermal therapy mediated by FA-IR780-NP effectively eradicated ovarian cancer tumors compared with other groups.

Conclusion: In this study, we present a potential, effective approach for ovarian cancer treatment through near-infrared fluorescence image-guided resection and photothermal therapy to eliminate malignant tissue.

Keywords: ovarian cancer, imaged-guide surgery, IR780 iodide, photothermal therapy, nanomedicine

\section{Introduction}

Ovarian cancer is the leading cause of gynecologic cancer deaths and is the country's fifth most common cause of cancer mortality in women. ${ }^{1}$ Due to the relatively asymptomatic nature of ovarian cancer and inadequate screening tests, approximately $75 \%$ of patients are diagnosed at late stage. ${ }^{2}$ Cytoreductive surgery and chemotherapy are the main treatments for ovarian cancer. ${ }^{3}$ Residual disease after surgery is confirmed to be important for patient survival, and the smaller the residual tumor $(\leq 1 \mathrm{~cm})$, the better the prognosis. ${ }^{4}$ However, it has been difficult to achieve optimal cytoreductive surgery ( $\leq 1 \mathrm{~cm}$ residuum) by relying on palpation and visual inspection by surgeons. ${ }^{5,6}$ Moreover, nearly $90 \%$ of patients who receive chemotherapy later develop chemotherapy resistance and inevitably succumb to their disease. ${ }^{7,8}$ Thus, new imaging techniques for early detection and the visualization of small tumors in surgery, as well as new adjuvant therapies, are urgently needed for patients with ovarian cancer. 
Over the past several years, intraoperative near-infrared (NIR) fluorescence imaging was developed as a novel technique for guiding surgeons in the detection and resection of residual malignant disease not detectable by conventional visual observation and palpation. ${ }^{9-11}$ Compared with traditional techniques (computed tomography [CT], magnetic resonance imaging [MRI], positron emission tomography [PET]/CT), NIR fluorescence imaging offers the benefits of high contrast and sensitivity, low cost, ease of use, and safety during surgery. ${ }^{12,13}$ Because of its high histocompatibility, indocyanine green (ICG) has been approved by the FDA for clinical application. ${ }^{14}$ However, the inability of ICG to bind specifically to tumor tissues results in a high rate of intraoperative false-positive images in patients with ovarian cancer. ${ }^{15}$ The current challenge for intraoperative NIR fluorescence imaging is the tumor-targeting sensitivity of NIR imaging contrast agent.

Loading an NIR dye onto a nanoparticle (NP) is an effective strategy to increase the tumor-targeting sensitivity of the contrast agent. The NP enables accumulation within the tumor matrix with an enhanced permeability and retention (EPR) effect due to the leakiness of the tumor vasculature combined with poor lymphatic drainage. ${ }^{16}$ In addition to the EPR effect, the NP surface is easily modified by tumor-targeting molecules to produce active targeting. ${ }^{17,18}$ Among the different drug delivery systems, lipid NPs are particularly promising because of their excellent biocompatibility and capacity to load large amounts of poorly soluble drugs as well as imaging probes. ${ }^{19}$ Over $90 \%$ of all epithelial ovarian cancers overexpress the folate(FA) receptor, and in ovarian cancers of serous morphology, this percentage is even higher (90$100 \%) .{ }^{20}$ Loading NIR fluorescence dye onto FA-modified NP combined with the passive targeting of EPR and the active targeting of FA may achieve an excellent tumortargeting effect in ovarian cancer.

IR780 iodide (IR780), an NIR dye, has been reported to have excellent NIR fluorescence ability, and due to its hydrophobicity, it could be easily loaded into liposomes. ${ }^{21}$ Moreover, IR780 as a photosensitizer upon NIR light irradiation can effectively convert light to heat, acting as photothermal therapy (PTT) and killing cancer cells. ${ }^{22}$ Compared with radiation therapy and chemotherapy, PTT has the advantages of free toxicity and convenience, ${ }^{23}$ making it an alternative adjuvant therapy during surgery. Due to its excellent NIR fluorescence imaging and photothermal conversion effect, there have been some studies on lipid nanosystems based on IR780 for cancer theranostics. Xue Yang et al reported a system comprising IR780 and sunitinib (an antiangiogenic drug) within liposomes (LipIR780-Sunitinib) to achieve antitumor angiogenesis and photothermal therapy in BALB/c mice bearing $4 \mathrm{~T} 1$ tumors. ${ }^{24}$ Guo Fang et al reported a smart nanocarrier loading IR780, doxorubicin, folate, and ammonium bicarbonate $(\mathrm{NH} 4 \mathrm{HCO} 3)$ (to generate $\mathrm{CO}_{2}$ bubbles) in liposomes (IR780-BTSL-FA) with a tumor-targeted accumulation effect and antitumor efficiency in an epidermoid carcinoma mouse model. ${ }^{25}$ Shihong $\mathrm{Li}$ et al described IR780-phospholipid micelles with tumor-specific NIRF imaging capacity in glioma mouse models, providing great potential for clinical imaging of brain tumors. ${ }^{26}$ Limited by the depth of light penetration, the nanosystems based on IR780-mediated PTT are not suitable for deep and large tumors; therefore, it is always used for superficial tumors and is combined with drug treatments. ${ }^{27,28}$ On the basis of these studies, we loaded IR780 into liposomes modified by FA (FA-IR780-NP) and applied it to ovarian cancer, a malignant tumor with high mortality located deep in the body, to evaluate whether it could be used in surgery to minimize residual lesions through NIR fluorescence imaging navigation and photothermal therapy. In this work, 1) the targeting ability of FA-IR780-NP was evaluated in vitro and in an ovarian xenograft tumor model, 2) the NIR fluorescence imaging ability in surgery was evaluated by fluorescence stereomicroscopy, and 3) the PTT effect of FA-IR780-NP was evaluated in vivo.

\section{Materials and methods}

\section{Materials}

1,2-Dipalmitoyl-sn-glycero-3-phosphocholine (DPPC), 1,2distearoyl-sn-glycero-3-phosphoethanolamine-N-[methoxy (polyethylene glycol)-2000] (DSPE-PEG(2000))and cholesterol were purchased from Avanti Polar Lipids, Inc. (Alabaster, AL, USA). DSPE-PEG(2000)-Folate was purchased from Nanocs Inc. (New York, NY, USA). IR780 iodide and ICG were purchased from Sigma-Aldrich (St. Louis, Missouri, USA). Cell Counting Kit-8 (CCK8) was purchased from Dojindo (Shanghai, China). 1,1 ${ }^{\text {` }}$ Dioctadecyl-3,3,3',3'-tetramethylindocarbocyanineperchlorate (DiI), 3,3'-dioctadecyloxacarbocyanine perchlorate (DiO), and 2-(4-amidinophenyl)-6-indolecarbamidine dihydrochloride (DAPI) were obtained from Beyotime Technology (Shanghai, China). Deionized water was 
obtained from a Millipore water purification system. All reagents were used without further purification.

\section{Synthesis of FA-IR780-NP}

FA-IR780-NP was generated by dissolving DPPC, cholesterol, DSPE-PEG (2000)-Folate, and IR780 at a weight ratio of 12:4:4:1 in $10 \mathrm{~mL}$ of chloroform in a flask. The solution was rotary evaporated in the dark to form a thin film. Then, $5 \mathrm{~mL}$ of distilled water was added to rehydrate the thin film. The hydrated lipid film was sonicated using a sonicator (Sonics \& Materials, Inc., Newtown, CT, USA) with an amplitude of $40 \%$ for $30 \mathrm{~min}$ and then extruded 21 times through a polycarbonate membrane with a pole size of $100 \mathrm{~nm}$ with a mini-extruder (Avanti Polar Lipids, Inc). ${ }^{29}$ NP loading IR780 (IR780-NP, without FA) and NP (without FA and IR780) were fabricated according to the same procedure. Finally, the collected NPs were freeze-dried using a freeze-drier (Bilon Instrument, Shanghai, China) and then stored at $-20{ }^{\circ} \mathrm{C}$ until further use.

\section{Characterization of FA-IR780-NP}

The size distribution of the NPs (FA-IR780-NP, IR780-NP, NP) was measured using a dynamic light scattering (DLS) analyzer (Malvern Instruments, Malvern, United Kingdom). The absorption spectra of free IR780, NP, IR780-NP, and FA-IR780-NP in dimethylsulfoxide (DMSO) or water were determined using a UV-Vis-NIR spectrophotometer (Shimadzu, Kyoto, Japan). The standard concentration curve was calculated based on the absorption peak of different concentrations of free IR780 $(0.5,1.0,1.5,2.0,2.5,3.0 \mu \mathrm{g} / \mathrm{mL})$ in DMSO. The dye loading efficiency of IR780 was calculated based on a standard concentration curve of free IR780:

Dye loading $(\%)=$ weight of the loaded dye/weight of the $\mathrm{NP} \times 100 \%$

Next, $8.0 \mathrm{mg}$ of ICG and IR780 were dissolved in $1 \mathrm{~mL}$ of DMSO and diluted to different concentrations $(4.0,2.0,1.0,0.5,2.5 \mathrm{e}-1,1.3 \mathrm{e}-1,6.3 \mathrm{e}-2,3.1 \mathrm{e}-2,1.6 \mathrm{e}-2$, 7.8e-3, 3.9e-3, 2.0e-3, 9.8e-4, 4.9e-4, 2.4e-4, 1.2e-4 mg/ $\mathrm{mL}$ ) in serum. FA-IR780-NP was directly diluted by serum $(1.3,6.7 \mathrm{e}-1,3.4 \mathrm{e}-1,1.7 \mathrm{e}-1,8.4 \mathrm{e}-2,4.2 \mathrm{e}-2,2.1 \mathrm{e}-2,1.0 \mathrm{e}-2$, $5.2 \mathrm{e}-3,2.6 \mathrm{e}-3,1.3 \mathrm{e}-3,6.5 \mathrm{e}-4,3.3 \mathrm{e}-4,1.6 \mathrm{e}-4,8.2 \mathrm{e}-5$ 4.1e$5 \mathrm{mg} / \mathrm{mL}$ ). Different concentrations of ICG, IR780 and FA-IR780-NP were added into black 96-well plates and detected using the in vivo IVIS imaging system (Perkin Elmer, Waltham, Massachusetts, USA) with a 780/845 nm excitation/emission wavelength filter.
Different concentrations of FA-NP-IR780 were added to 96-well plates and exposed to NIR light $(808 \mathrm{~nm}$, $\left.1.0 \mathrm{~W} / \mathrm{cm}^{2} 5 \mathrm{~min}\right)$. The temperature changes were monitored using an infrared thermal imaging camera (Jetyoo Instruments, Shanghai, China)

\section{Cell lines and culture}

SKOV3 cells were purchased from ProCell Company (Wuhan, China) and cultured in the ATCC-recommended media McCoy's 5A with 10\% FBS (PAN-Biotech, Aidenbach, Germany) and $1 \%$ penicillin/streptomycin and incubated at $37{ }^{\circ} \mathrm{C}$ with $5 \% \mathrm{CO}_{2}$. At approximately $80 \%$ confluence, the cells were passaged at a ratio of $1: 2$.

\section{Toxicity in vitro}

SKOV3 cells were separately cultured in 96-well plates at a density of $5 \times 10^{3}$ cells $/ \mathrm{mL}$ in each well. After $24 \mathrm{~h}$ of culture, the cells were washed 2 times with PBS, and then $100 \mu \mathrm{L}$ of medium containing different concentrations of FA-IR780-NP $(0 \mu \mathrm{g} / \mathrm{mL} ; 5 \mu \mathrm{g} / \mathrm{mL}, 10 \mu \mathrm{g} / \mathrm{mL}, 20 \mu \mathrm{g} / \mathrm{mL}$, $30 \mu \mathrm{g} / \mathrm{mL}, 40 \mu \mathrm{g} / \mathrm{mL} ; \mathrm{n}=6$ per group) was added to incubate with the cells for $24 \mathrm{~h}$. Next, CCK- 8 was used to detect cell viability. Briefly, $10 \mu \mathrm{L}$ of CCK- 8 solution was added to each well and incubated for $1 \mathrm{~h}$. Then, the absorbance was recorded at $450 \mathrm{~nm}$ using a microplate reader (ELX800, Bio Tek, Winooski, VT, USA).

To evaluate therapeutic effects in vitro, after incubation with different concentrations of FA-IR780-NP for $24 \mathrm{~h}$, SKOV3 cells were exposed to NIR laser $(808 \mathrm{~nm}$, $1 \mathrm{~W} / \mathrm{cm}^{2}$ ) for $3 \mathrm{~min}$. Cell viability was determined using the CCK-8 assay.

\section{Assessment of target binding in vitro}

SKOV3 cells were separately cultured in confocal dishes at a density of $5 \times 10^{4}$ cells $/ \mathrm{mL}$ in each well. After $24 \mathrm{~h}$ of culture, the cells were washed 2 times with PBS; then, medium containing $40 \mu \mathrm{g} / \mathrm{mL}$ different DiI-labeled NPs (DiI-FA-IR780-NP, DiI-IR780-NP, or DiI-NP) was added ( $n=3$ per group). After $2 \mathrm{~h}$ of incubation, the cells were washed three times with PBS, fixed in 4\% paraformaldehyde for $15 \mathrm{~min}$, and then stained with DAPI for the nucleus and $\mathrm{DiO}$ for the cytomembrane. The stained cells were imaged using a laser confocal scanning microscopy (LCSM, Nikon, Tokyo, Japan). Moreover, the binding efficiency between cells and NPs ( $n=3$ per group) was determined using flow cytometry (FCM; FACSVantage; BD; Franklin Lake, NJ, USA) after trypsinization. 


\section{Toxicity in vivo}

All animal studies were approved and performed in accordance with animal ethics committee of Chongqing Medical University and the national guidelines on the care and use of laboratory animals. Six- to eight-weekold female $\mathrm{BALB} / \mathrm{c}$ mice were randomly grouped and treated once with different doses of FA-IR780-NP (5.5, $17.5,55 \mathrm{mg} / \mathrm{kg}$ ) administered intravenously ( $\mathrm{n}=6$ per group). The control group received $200 \mu \mathrm{L}$ of PBS. The survival rates of the animals were observed 2 weeks after administration. At $14 \mathrm{~d}$, the mice were sacrificed, and the organs (hearts, livers, spleens, lungs and kidneys) were harvested for hematoxylin-eosin (H\&E) staining.

\section{Animal model}

$\mathrm{BALB} / \mathrm{c}$ female nude mice were subcutaneously injected with SKOV3 cells $\left(2 \times 10^{6}\right.$ cells in $100 \mu \mathrm{L}$ of PBS $)$ on the back. Tumor sizes were assessed every $5 \mathrm{~d}$ with a caliper using the following formula: $0.5 \times$ width $^{2} \times$ length. When the tumor size reached approximately $100 \mathrm{~mm}^{3}$, the mice were used for further experiments.

\section{NIR fluorescence and biodistribution in vivo}

Mice with xenograft tumors were randomly divided into three groups: free IR780, IR780-NP, and FA-IR780-NP groups ( $\mathrm{n}=3$ per group). After the intravenous injection of free IR780 (1 mg/kg), IR780-NP (30 mg/kg; the corresponding IR780 dose was $1 \mathrm{mg} / \mathrm{kg}$ ) or FA-IR780-NP (30 mg/kg; the corresponding IR780 dose was $1 \mathrm{mg} / \mathrm{kg}$ ), the mice were imaged using the in vivo IVIS imaging system at the indicated time points (preinjection, and at $1,2,3$ and $5 \mathrm{~d}$ postinjection) under anesthesia with pentobarbital sodium salt. The mice were sacrificed at $5 \mathrm{~d}$ postinjection, and the major organs, including the hearts, livers, spleens, lungs, and kidneys, and tumors were dissected for imaging. The corresponding fluorescence intensities were analyzed by IVIS software.

\section{Intraoperative navigation for tumors in vivo}

$\mathrm{BALB} / \mathrm{c}$ female nude mice harboring xenografts of SKOV3 tumors were intravenously injected with $30 \mathrm{mg} /$ $\mathrm{kg}$ FA-IR780-NP. The mice were anesthetized at $3 \mathrm{~d}(\mathrm{n}=3$ per group), and the skin overlying the tumor was removed. Surgical areas were imaged in real-time using a fluorescence stereomicroscope (Nikon AZ100, Nikon). The NIR filter cube (Chroma Technology Corp, Bellows Falls, VT, USA) was a $710 / 75 \mathrm{~nm}$ exciter and $810 / 90 \mathrm{~nm}$ emitter (acquisition time: $10 \mathrm{~s}$ ).

To further investigate the ability of FA-IR780-NP to distinguish tumors and adjacent normal tissues at the microscopic scale, BALB/c female nude mice harboring SKOV3 tumor xenografts ( $\mathrm{n}=3$ per group) were intravenously injected with FA-NP-IR780 (30 mg/kg) labeled with DiI. After $3 \mathrm{~d}$, the tumors and the adjacent tissues were removed for pathological examination. The pathological sections were observed under fluorescence and white light through an inverted fluorescence microscope (Olympus, Tokyo, Japan).

\section{Therapeutic evaluation of FA-IR780-NP in vivo}

When the tumor size reached $\sim 100 \mathrm{~mm}^{3}$, the tumor-bearing mice were intravenously administered PBS $(200 \mu \mathrm{L}$; as a control), free IR780 (1 mg/kg), IR780-NP (30 mg/kg; the corresponding IR780 dose was $1 \mathrm{mg} / \mathrm{kg}$ ) or FA-IR780-NP (30 $\mathrm{mg} / \mathrm{kg}$; the corresponding IR780 dose was $1 \mathrm{mg} / \mathrm{kg}$ ) ( $\mathrm{n}=6$ per group). At $3 \mathrm{~d}$ after administration, the tumor regions were irradiated by an $808 \mathrm{~nm}$ NIR laser $(1.0 \mathrm{~W} /$ $\mathrm{cm}^{2}$ ) for $10 \mathrm{~min}$ under anesthetization. The temperature was monitored by an infrared thermal imaging camera.

Next, the tumor-bearing mice were randomly divided into four groups ( $\mathrm{n}=6$ per group): control, FA-IR780-NP, laser-only, and PTT (FA-IR780-NP mediated photothermal therapy). The mice in the control group were intravenously administered $200 \mu \mathrm{L}$ of PBS. The mice in the FA-IR780$\mathrm{NP}$ group were intravenously administered $30 \mathrm{mg} / \mathrm{kg}$ FAIR780-NP. For the NIR laser group, the tumor area was irradiated using an $808 \mathrm{~nm}$ NIR laser $\left(1 \mathrm{~W} / \mathrm{cm}^{2}\right)$ for 10 min under anesthetization with pentobarbital sodium salt. The mice in the PTT group were intravenously administered $30 \mathrm{mg} / \mathrm{kg}$ FA-NP-IR780 through the tail; at $3 \mathrm{~d}$ after administration, the tumor areas were irradiated using an $808 \mathrm{~nm}$ laser $\left(1 \mathrm{~W} / \mathrm{cm}^{2}\right)$ for $10 \mathrm{~min}$ under anesthesia with pentobarbital sodium salt. The temperature was monitored using an infrared thermal imaging camera. Tumor volumes and mice survival were monitored for $30 \mathrm{~d}$ after treatments. The tumor weights were measured for two weeks after treatments. Tumor and major organs were dissected at $24 \mathrm{~h}$ after treatments ( $\mathrm{n}=3$ per group) and then fixed with $4 \%$ polyoxymethylene for histological analysis (H\&E, terminal-deoxynucleotidyl transferase mediated dUTP nick-end labeling [TUNEL], proliferating cell nuclear antigen [PCNA]). 


\section{Statistical analysis}

All statistical evaluations were performed with one-way analysis of variance (ANOVA). Statistical analyses were performed using SPSS 22.0 software, and significance was considered at $P<0.05$.

\section{Results and discussion}

\section{Characterization of FA-NP-IR780}

IR780, despite its optical advantages, is hydrophobic, which limits its potential application in vivo. Encapsulation of IR780 into liposomes could increase its biocompatibility. ${ }^{30}$ As shown in Figure 1A, due to the loading of IR780 and FA, FA-IR780-NP appeared yellow-green, while NP was white, and NP-IR780 was green under natural light. There was no precipitation or aggregation, and the NPs dispersed well in aqueous solution. The sizes of NP, IR780-NP and FA-IR780-NP were $160.30 \pm 81.99 \quad \mathrm{~nm} \quad(\mathrm{PDI}=0.171), \quad 154.20 \pm 47.70 \quad \mathrm{~nm}$ $(\mathrm{PDI}=0.183)$ and $156.50 \pm 65.91 \mathrm{~nm}(\mathrm{PDI}=0.178)$, respectively (Figure 1B). To detect the encapsulation efficiency and dye loading of IR780, a UV-Vis-NIR spectrophotometer was applied. Free IR780 exhibited an absorbance peak at $793 \mathrm{~nm}$ (Figure 1C), and the absorbance peak intensity increased linearly with increased concentrations
(Figure 1D). As shown in Figure 1E, the packing of IR780 in the NPs resulted in a red shift of the absorption spectrum in water compared with that of free IR780, and the absorption peak of FA-IR780-NP was obviously lower than that of IR780-NP. After being dissolved in DMSO, the structures of NPs were destroyed, and the IR780 was free (Figure 1F). According to the calibration curve of IR780 in DMSO (Figure 1D), the dye loading in IR780$\mathrm{NP}$ and FA-IR780-NP was calculated to be approximately $3.87 \pm 0.26 \%$ and $3.64 \pm 0.33 \%$, respectively, with no significant difference in dye loading between the two types of NPs $(P>0.05)$.

Next, the NIR fluorescence imaging ability of FAIR780-NP was evaluated. First, the fluorescence imaging ability of IR780 was compared with that of ICG. Specifically, previous studies have confirmed that the fluorescence intensity of IR780 is higher in serum than that in water. ${ }^{31}$ To mimic the in vivo environment, various concentrations of IR780 and ICG were dispersed in serum. As shown in Figure 2A, the fluorescence signal of IR780 was significantly stronger than that of ICG. Moreover, at lower concentrations of both ICG and IR780, the fluorescence intensity increased with the concentration, while at higher concentrations, the fluorescence intensity decreased, which indicated a self-quenching phenomenon. ${ }^{32}$ Therefore, the
A

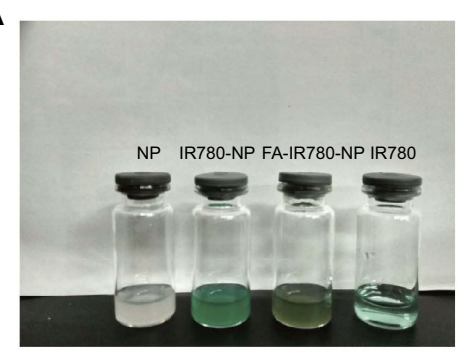

D

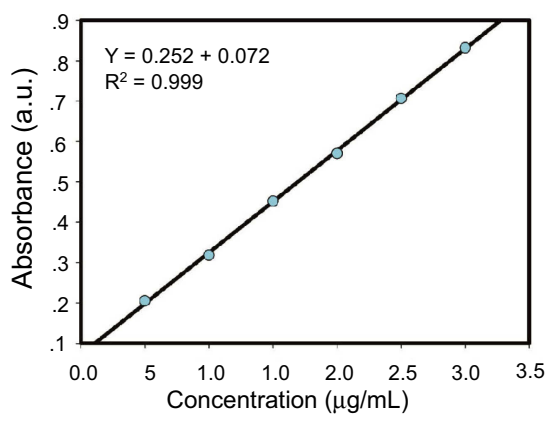

B
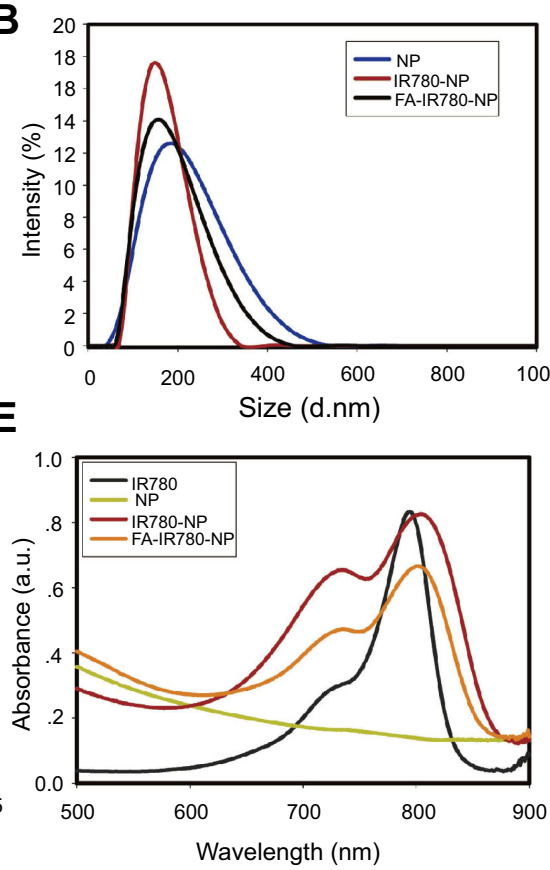

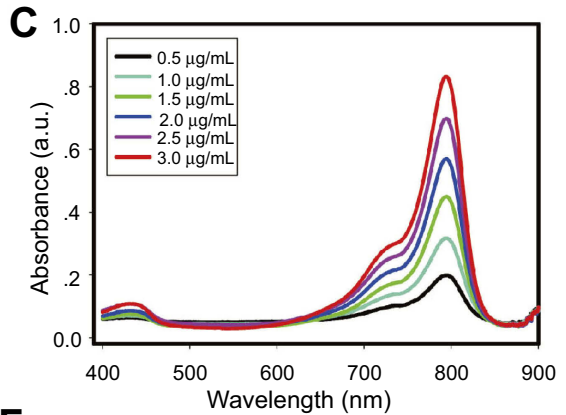

$F$

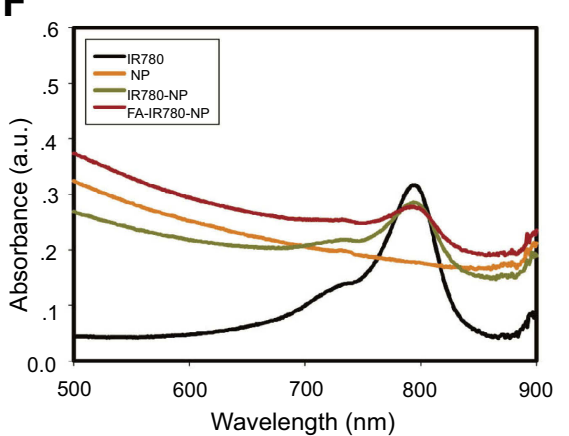

Figure I Features of NPs. (A) Images of NP, IR780-NP, FA-IR780-NP, and IR780 under natural light. (B) Size distributions of NP, IR780-NP, and FA-IR780-NP. (C) UV-VisNIR absorbance spectra of IR780 at elevated concentrations in DMSO. (D) Linear relationship between absorbance peak intensity and IR780 concentrations. (E) UV spectra of IR780 and $60 \mu \mathrm{g} / \mathrm{mL}$ NPs (NP, IR780-NP, and FA-IR780-NP) dissolved in water. (F) UV spectra of IR780 and I5 $\mu \mathrm{g} / \mathrm{mL}$ NPs (NP, IR780-NP, and FA-IR780-NP) dissolved in DMSO. The values are expressed as the mean $\pm \mathrm{SD}, \mathrm{n}=3$ per group. 
A

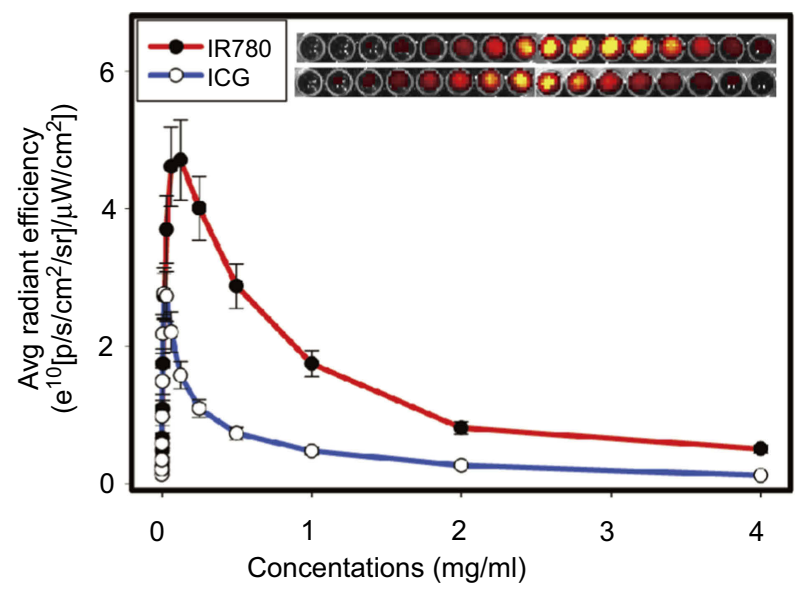

B

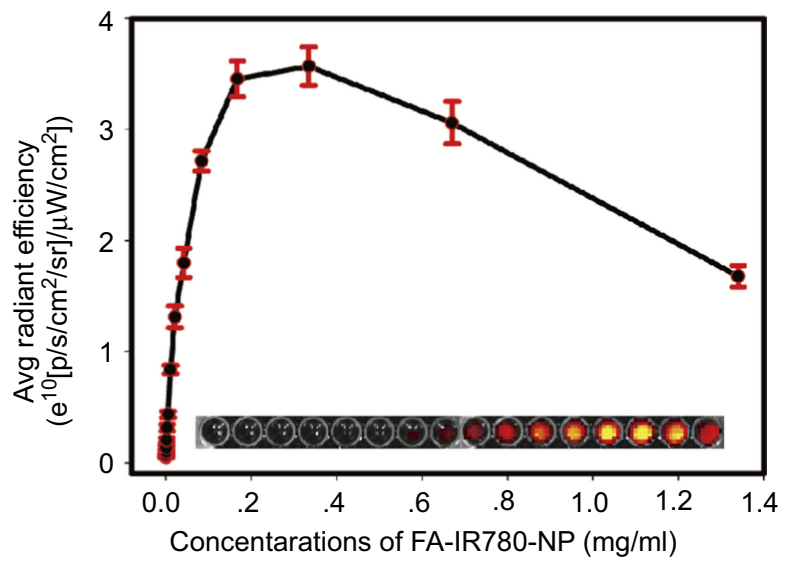

C

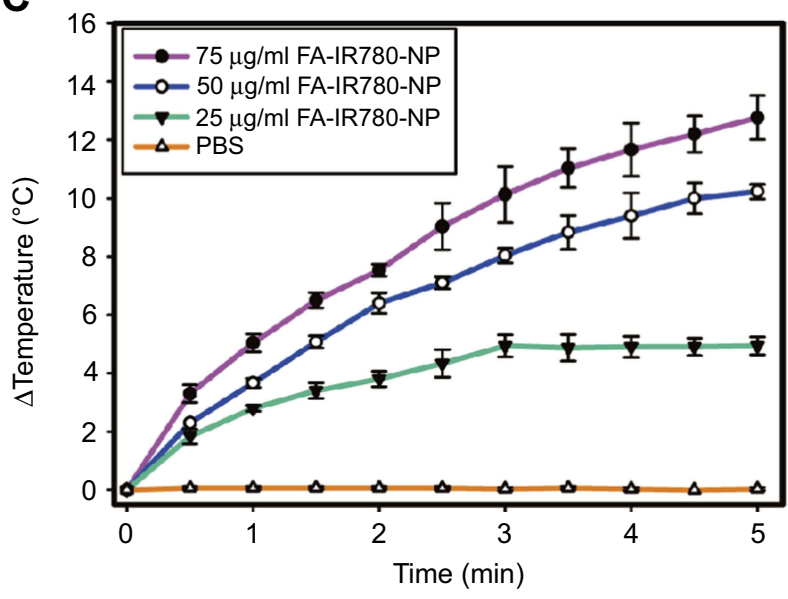

Figure 2 Fluorescence and photothermal property of FA-IR780-NP in vitro. (A) Comparison of the in vitro fluorescence imaging and corresponding intensities of ICG and IR780 at different concentrations. (B) In vitro fluorescence imaging and corresponding intensities of FA-IR780-NP at different concentrations. (C) In vitro temperature changes of PBS and different concentrations of NP-IR780 under laser irradiation $\left(808 \mathrm{~nm}, 1.0 \mathrm{~W} / \mathrm{cm}^{2}, 5 \mathrm{~min}\right)$. The values are expressed as the mean \pm SD, $\mathrm{n}=3 \mathrm{per}$ group.

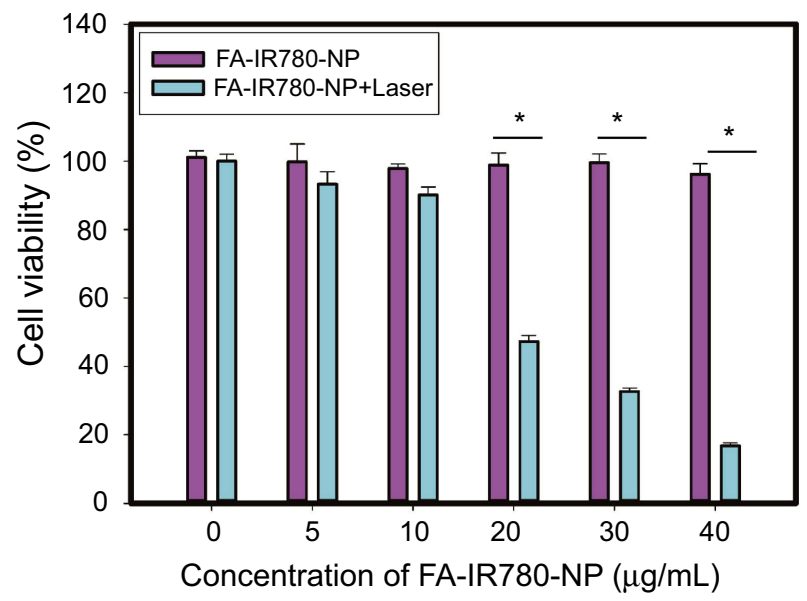

Figure 3 SKOV3 cell viability after incubation with various concentrations of FA-IR780-NP for $24 \mathrm{~h}$, followed by NIR laser irradiation for $3 \mathrm{~min}(808 \mathrm{~nm}$, I $\left.\mathrm{W} / \mathrm{cm}^{2}\right)(* P<0.05)$. The values are expressed as the mean $\pm S D, n=6$ per group. hydrophobicity of IR780 with higher loading and a stronger fluorescence signal may be more suitable for loading onto NP for in vivo imaging.

The NIR fluorescence imaging ability of FA-IR780-NP was evaluated, and FA-IR780 showed a similar fluorescence phenomenon to that of IR780 (Figure 2B). Next, the ability of FA-IR780-NP to act as a photosensitizer and convert NIR light energy into heat upon NIR light was detected. As the concentration increased, the temperature increased faster and to a higher degree (Figure 2C).

\section{Tumor cell toxicity and targeting ability of FA-IR780-NP}

FA-IR780-NP has been demonstrated to exhibit NIR fluorescence imaging ability and photothermal conversion in vitro. Next, the cytotoxicity of PTT mediated by FA- 

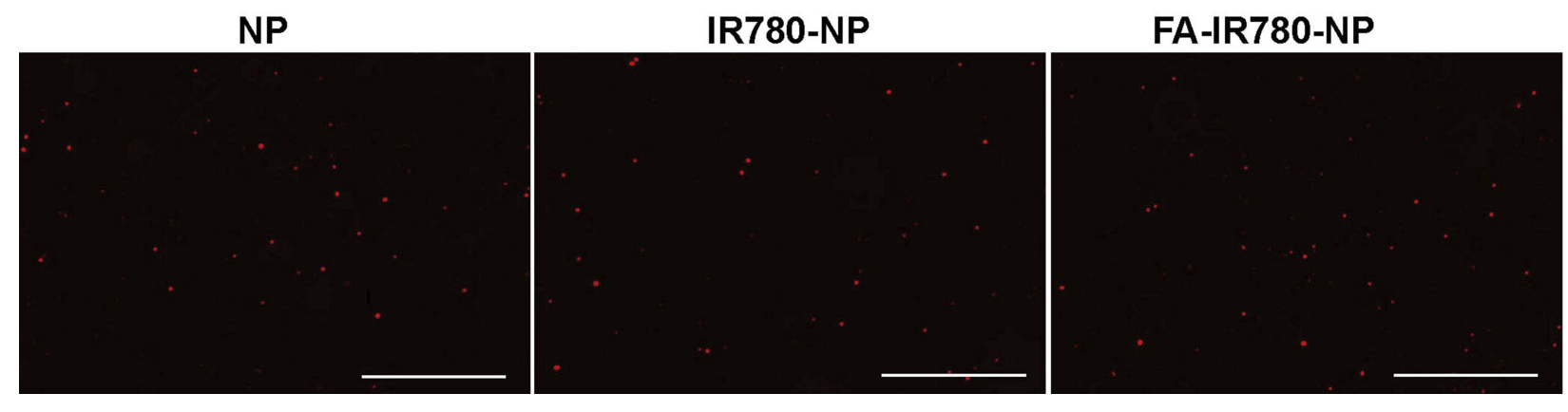

Figure 4 Fluorescence images of NP, IR780-NP, and FA-IR780-NP labeled with Dil. The scale bars are $5 \mu \mathrm{m}$.

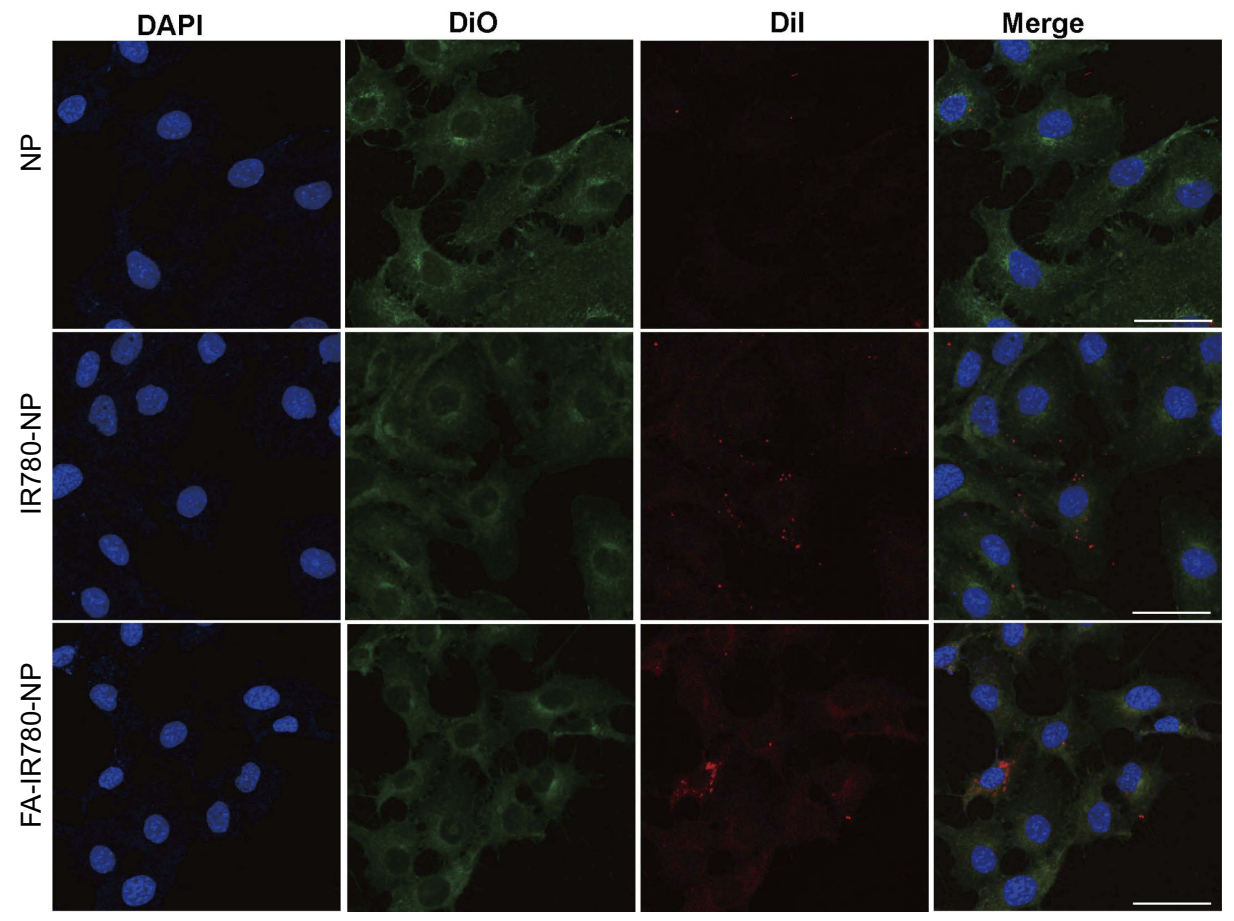

Figure 5 Intracellular uptake of different NPs (NP, IR780-NP, FA-IR780-NP) by confocal laser fluorescence microscopy after $2 \mathrm{~h}$ of incubation. Blue (DAPI) represents cell nuclei; green (Dio) reveals the cytomembrane; whereas the red (Dil) dots imply Dil-labeled NPs. The scale bars represent $20 \mu \mathrm{m}, \mathrm{n}=3$ per group.

IR780-NP was investigated using a CCK-8 assay. A 24-h incubation of FA-IR780-NP $(5 \mu \mathrm{g} / \mathrm{mL}$ to $40 \mu \mathrm{g} / \mathrm{mL})$ exhibited negligible toxicity against SKOV3 cell viability. However, under the irradiation of NIR laser $(808 \mathrm{~nm}$, $1 \mathrm{~W} / \mathrm{cm} 2,3 \mathrm{~min}^{2}$ ), the cells exhibited concentrationdependent toxicity (Figure 3 ).

Excellent tumor targeting of NPs is the key for imaging and therapy of cancer; therefore, the targeting ability of NPs was evaluated in vitro. Three kinds of NPs (NP, IR780-NP, FA-IR780-NP) were labeled with DiI (Figure 4) and coincubated with SKOV3 cells for $2 \mathrm{~h}$. As shown in Figures 5 and $\mathrm{S} 1$, more NPs entered the cells in the FA-IR780-NP group than in the NP and IR780-NP groups. Moreover, quantitative analysis by FCM showed that the binding efficiencies of the
FA-IR780-NP, IR780-NP and NP groups were $94.78 \pm 4.19 \%$, $77.75 \pm 2.33 \%$, and $45.26 \pm 9.57 \%$, respectively (Figure 6). The FA-IR780-NP group exhibited higher binding efficiency than the IR780-NP and NP groups. Particularly, IR780-NP also showed a higher binding efficiency than NP, which indicated that IR780 improved the tumor-targeting ability of IR780-NP in vitro. The tumor-specific binding of FAIR780-NP leads to a high accumulation in tumors and may be beneficial for NIR fluorescence imaging and PTT.

\section{Toxicity of FA-IR780-NP in vivo}

The photothermal properties, NIR fluorescence ability and targeting properties of FA-IR780-NP to SKOV3 cells have been demonstrated in vitro. Thus, the in vivo translation 

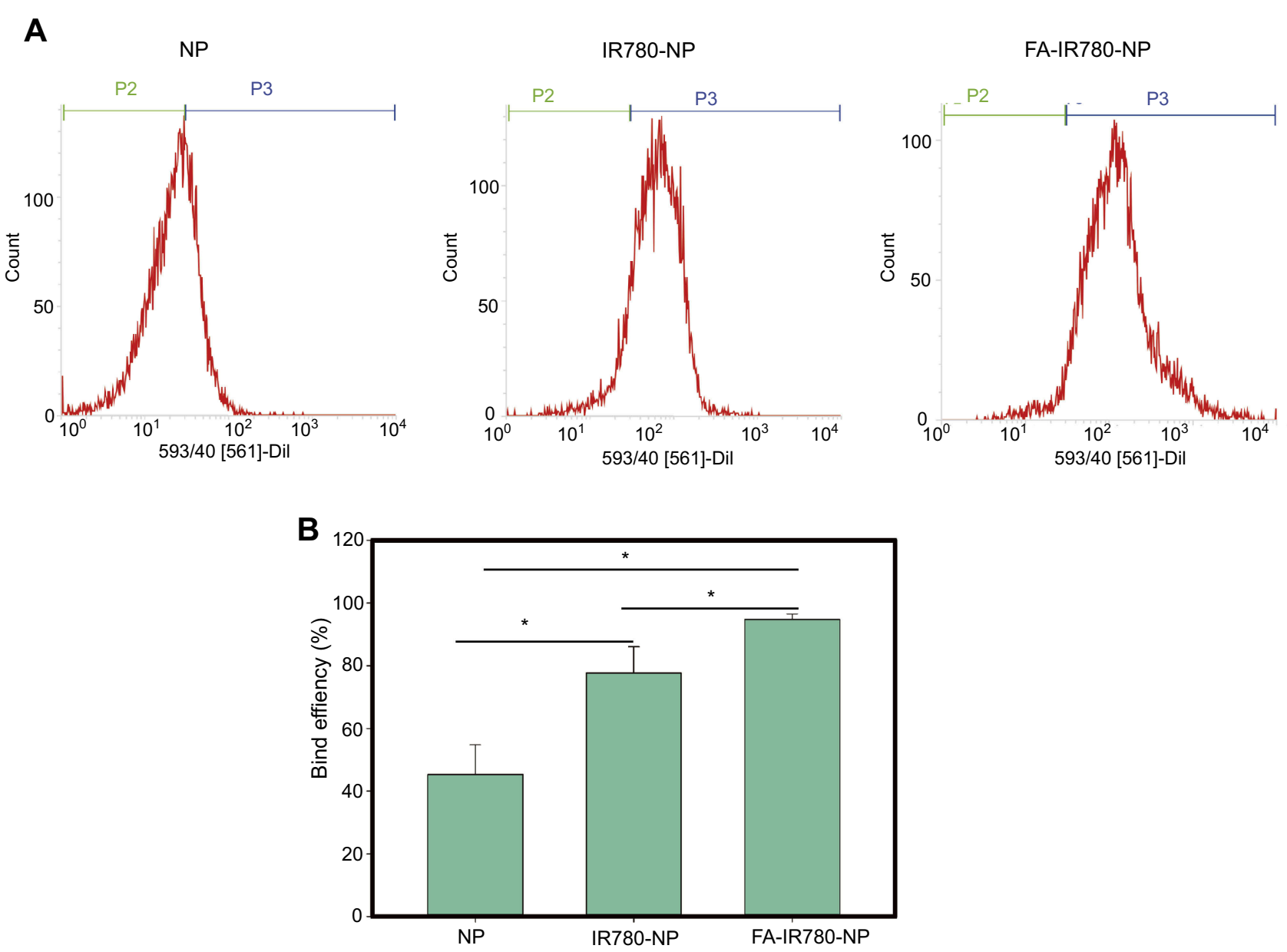

Figure 6 Cell binding efficiency was detected by FCM in various groups. (A) Binding efficiency of different NPs (NP, IR780-NP, FA-IR780-NP) with SKOV3 cells by FCM after $2 \mathrm{~h}$ of incubation. (B) Binding efficiency rate in various groups $(* P<0.05)$. The values are expressed as the mean $\pm S D, n=3$ per group.

potential needed to be evaluated. After the intravenous administration of FA-IR780-NP at different doses (5.5, $17.5,55 \mathrm{mg} / \mathrm{kg}$ ), no obvious abnormal behaviors were observed in any of the mice during two weeks of observation. Compared with the control group, the FA-IR780-NP groups showed negligible fluctuations of H\&E staining of the main organs (Figure 7). These results indicated that a dose of FA-IR780-NP below $55 \mathrm{mg} / \mathrm{kg}$ caused no significant toxicity during the testing period in vivo.

\section{NIR fluorescence and biodistribution in vivo}

To validate FA-IR780-NP for in vivo ovarian cancer NIR fluorescence imaging, mice with subcutaneous SKOV3 tumors were used to evaluate tumor targeting and organ distributions. After the mice were intravenously administered IR780 (1 mg/kg), IR780-NP (30 mg/kg; the corresponding IR780 dose was $1 \mathrm{mg} / \mathrm{kg}$ ) and FA-IR780-NP
(30 $\mathrm{mg} / \mathrm{kg}$; the corresponding IR780 dose was $1 \mathrm{mg} / \mathrm{kg}$ ), fluorescence imaging was performed at different time points. As shown in Figure 8A, FA-IR780-NP showed better targeting ability to SKOV3 tumors than did IR780NP and IR780. Free IR780 was shown to accumulate in SKOV3 tumors from the third day after injection, which indicated the binding of IR780 to SKOV3 tumors. The IR780-NP group also showed tumor targeting, but a considerable fluorescence signal was apparent in other normal tissues at $5 \mathrm{~d}$. From the second day, the fluorescence intensity in tumor regions of the FA-IR780-NP group was higher than that of other groups (Figure 8B). Particularly, two days after the injection, the whole body of the mice showed strong fluorescence, and the maximum fluorescence value of the tumor areas in IR780-NP and FA-IR780-NP groups were delayed to $48 \mathrm{~h}$, which was different from the conventional $\sim 20 \mathrm{~h}$ of NPs. ${ }^{33}$ This phenomenon may be related to self-quenching as observed 


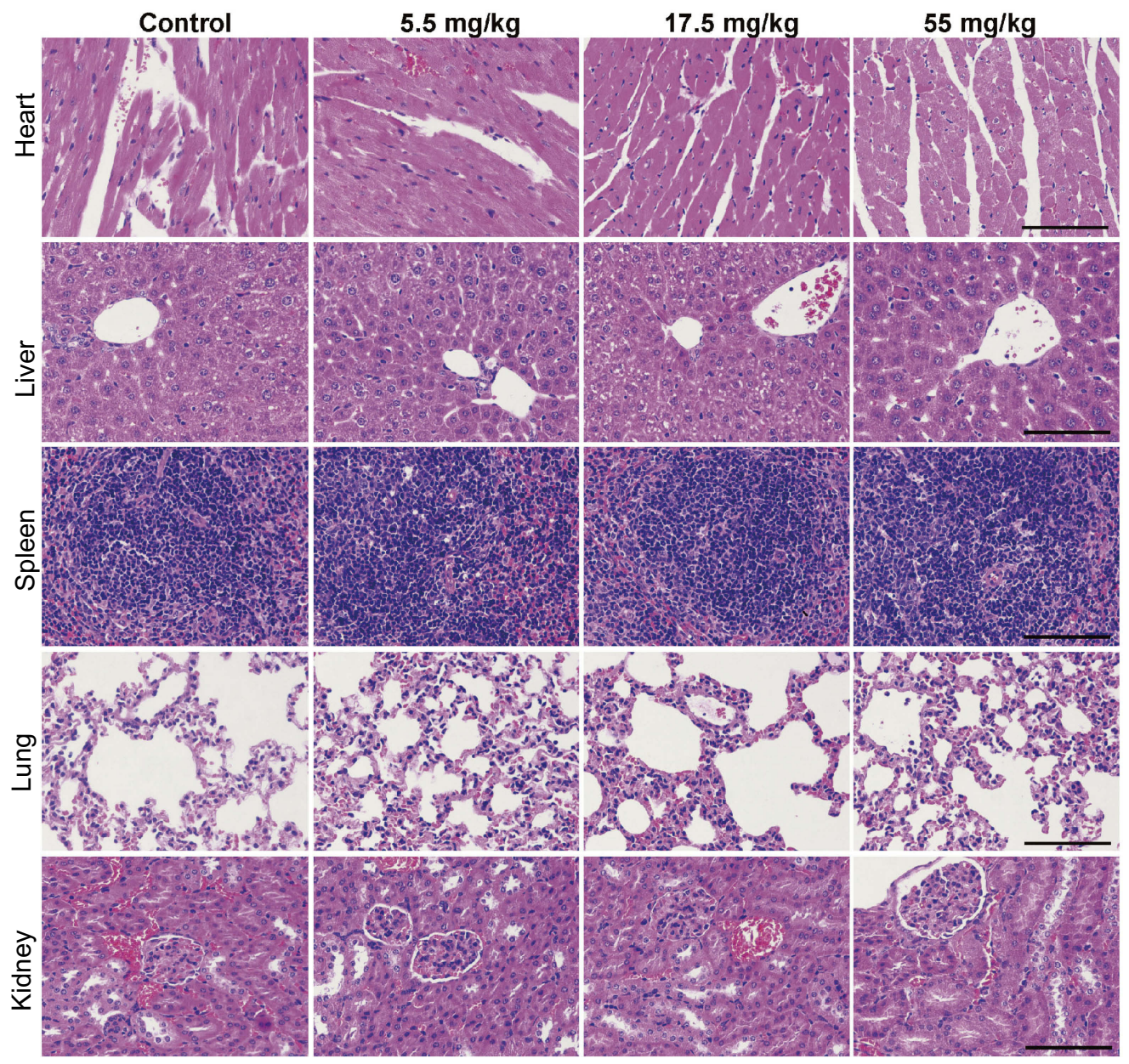

Figure 7 Organ tissues of mice were stained with H\&E after $14 \mathrm{~d}$ of treatment with PBS or $5.5 \mathrm{mg} / \mathrm{kg}$ or $17.5 \mathrm{mg} / \mathrm{kg}$ or $55 \mathrm{mg} / \mathrm{kg}$ FA-IR780-NP. The scale bars represent $100 \mu \mathrm{m}, \mathrm{n}=6$ per group.

in vitro. In other words, the fluorescence intensity of IR780 would not decrease even if the IR780 concentration decreased within a certain range.

A tumor-to-background fluorescence signal intensity ratio (TBR) is a key factor for intraoperative imaging, ${ }^{9,32}$ therefore, the ratio of the mean fluorescence signal intensity (MFI) of the tumor region to that of the surrounding adjacent tissue (considered background fluorescence) was calculated. As shown in Figure 8C, the TBRs of all groups gradually increased over time, and the TBR of FA-IR780-NP was higher than that of the other groups from the third day.

To further assess the distribution of IR780, IR780-NP, and FA-IR780-NP in vivo, tumors and major organs (hearts, livers, spleens, lungs, and kidneys) were collected and imaged at $5 \mathrm{~d}$ postinjection. The obtained images revealed enhanced fluorescence signals in SKOV3 tumors, demonstrating an effective accumulation of IR780, IR780-NP and FA-IR780-NP (Figure 8D). Moreover, compared with free IR780, the higher fluorescence signal intensity in the tumors, livers and lungs in the IR780-NP group indicated that loading a dye onto NPs significantly prolonged their circulation time and changed their distribution in the body, which was related to the EPR effect. ${ }^{34}$ The addition of FA improved the tumor-targeting effect of NP, and combined with the prolonged circulation, FA-IR780-NP showed superior fluorescence imaging capability for tumors than the other groups did (Figure 8E). Therefore, the excellent tumor-targeting ability of FA-IR780-NP was attributed to the EPR effect of NP, the active targeting of folate and the selective accumulation in tumors of IR780.

\section{Intraoperative navigation for tumors in vivo}

Motivated by the excellent tumor-target effect of FAIR780-NP on SKOV3 tumors in vivo through an IVIS fluorescence imaging system, intraoperative imaging- 
A

A Pre $1 \mathrm{~d}$

0
$\infty$
$\stackrel{\underline{x}}{\alpha}$

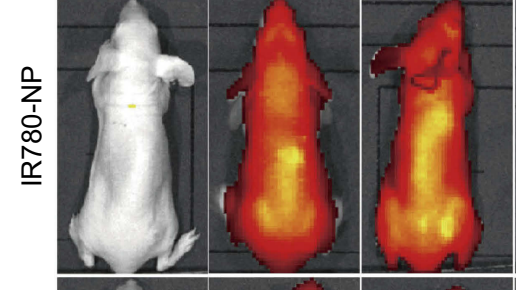

re
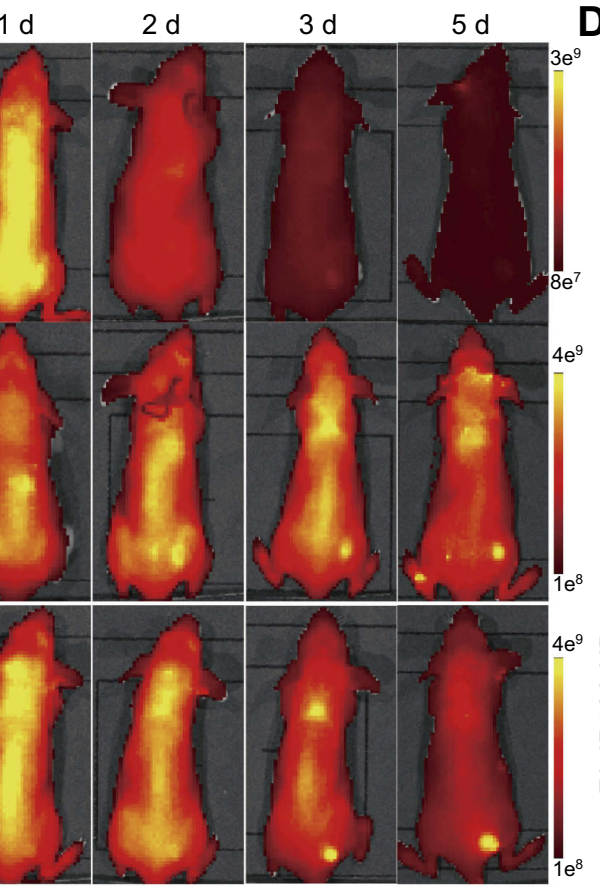

C

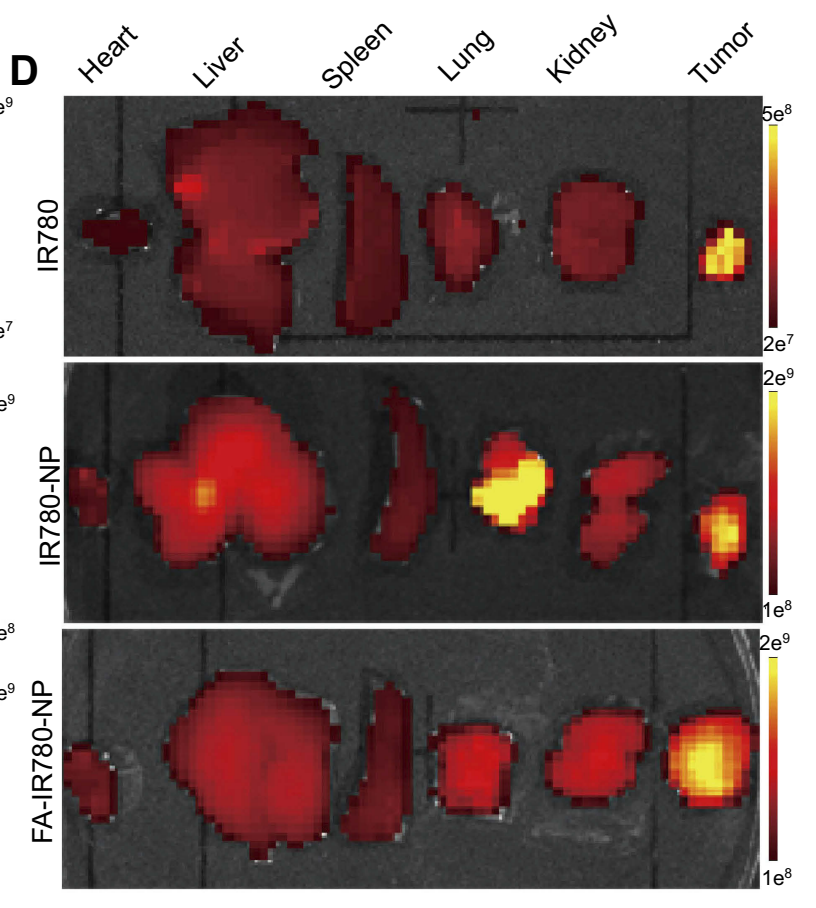

E
B

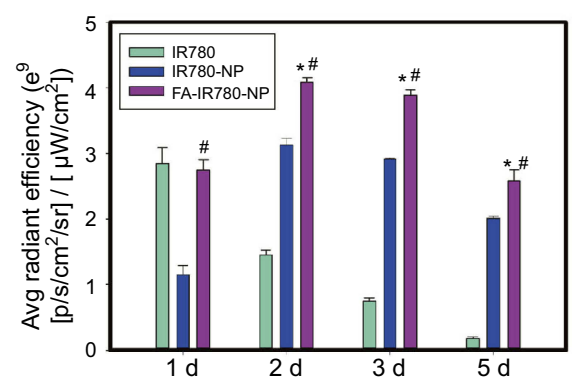

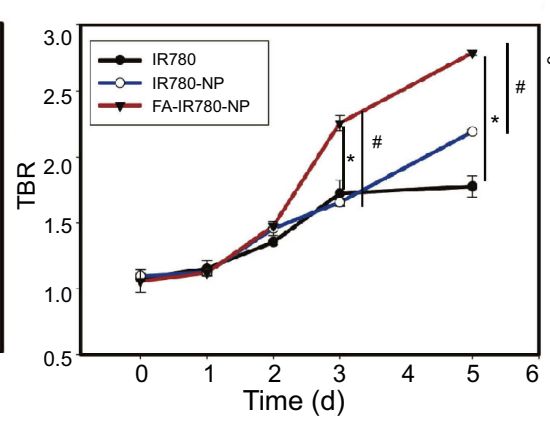

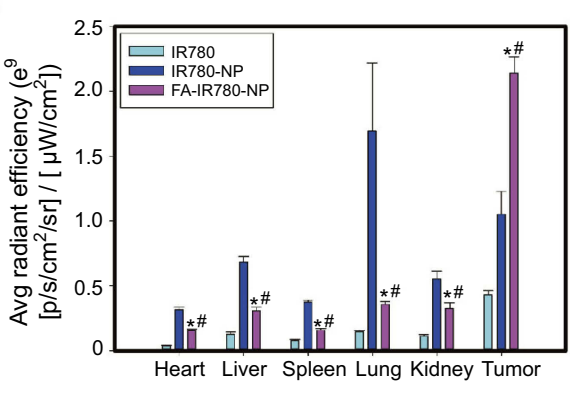

Figure 8 FA-IR780-NP was demonstrated to have excellent tumor-targeting ability in vivo. (A) NIR fluorescence imaging of tumor-bearing mice after the intravenous administration of free IR780, IR780-NP, or FA-IR780-NP at different time points (pre, I d, $2 \mathrm{~d}, 3 \mathrm{~d}, 5 \mathrm{~d}$ ). (B) Fluorescence signal intensities within tumor regions at the corresponding time points of A. (C) TBRs of IR780, IR780-NP and FA-IR780-NP at the different time points. (D) Ex vivo fluorescence imaging of mouse major organs and tumor nodes at $5 \mathrm{~d}$ postinjection and (E) corresponding fluorescence signal intensities. The values are expressed as the mean \pm SD, $n=3$ per group. $* P<0.05$ when the FAIR780-NP group was compared with the IR780 group. ${ }^{\#} P<0.05$ when the FA-IR780-NP group was compared with the NP-IR780 group.

guided cancer surgery was evaluated. Considering the TBR and the fluorescence signal intensity in the tumor, intraoperative navigation was performed on the third day after FA-IR780-NP administration. The mice bearing SKOV3 tumors underwent surgery, and the tumors were exposed to white light and NIR light for imaging. As shown in Figure 9A, the white light image of the tumor and fluorescence signals generated by FA-NP-IR780 strongly overlapped; notably, the tumor's diameters were less than $1 \mathrm{~cm}$. Moreover, the tumor size shown by NIR fluorescence was larger than that shown by white light visual inspection, which would be useful for surgeons to identify the boundary of malignant tissues intraoperatively, because only relying on the experience of surgeons to identify the tumor boundary could lead to positive margins. ${ }^{3}$ The mean fluorescence intensities in the tumor regions were significantly higher than that in the adjacent tissues (Figure 9B). During the operation, the tumors and the adjacent tissues were excised for pathological examination. As expected, the malignant tissues had a higher fluorescence signal than the adjacent normal tissues (Figure 9C). H\&E staining further confirmed that the fluorescence tissues were indeed cancerous lesions (Figure 9D). These results indicated that FA-IR780-NP efficiently delineates the boundaries of ovarian tumors during surgery. 
A

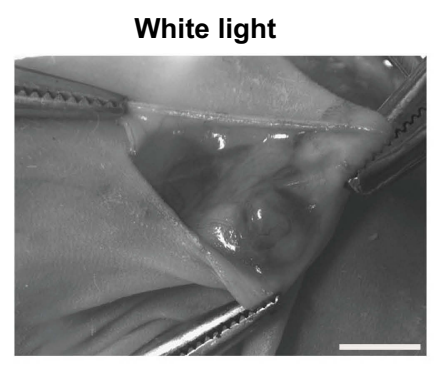

B

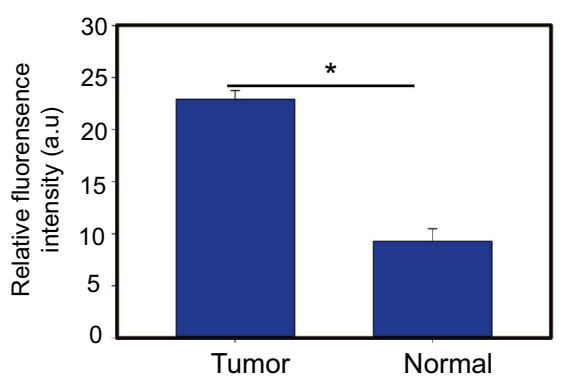

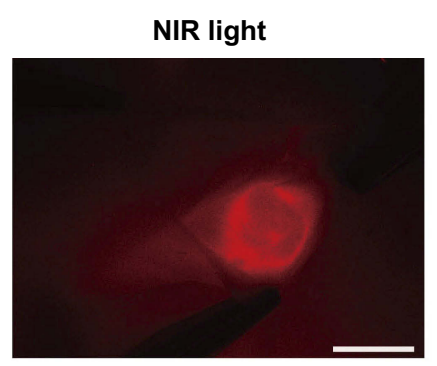

C

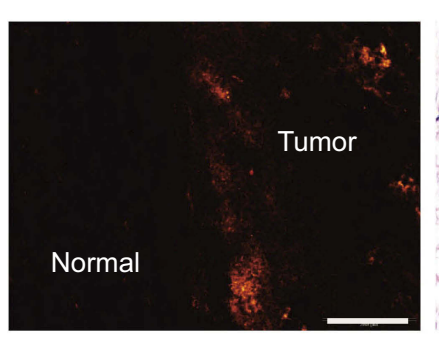

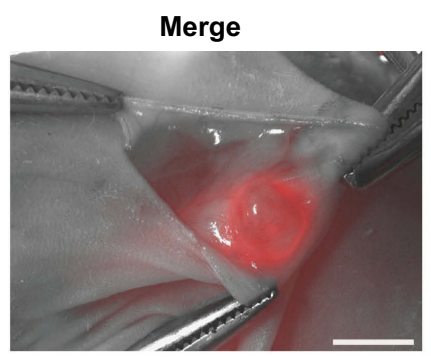

D

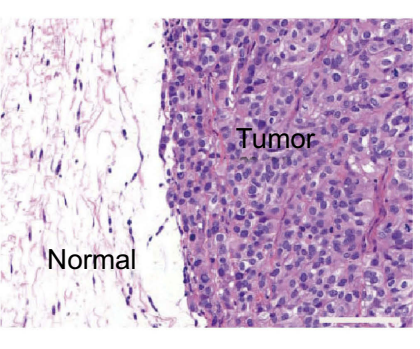

Figure 9 Intraoperative navigation for SKOV3 tumors by FA-IR780-NP in vivo. (A) Intraoperative real-time monitoring by a fluorescence stereomicroscope on the third day after the administration of FA-IR780-NP. The bars represent $2000 \mu \mathrm{m}$, and the acquisition time was $10 \mathrm{~s}$. (B) The mean fluorescence intensity of the tumor and adjacent normal tissues in panel $A(* P<0.05)$. (C) Fluorescence image of the tumor and normal tissue cross-sections. The bar represents $200 \mu \mathrm{m}$, and the acquisition time was $5 \mathrm{~s}$. (D) H\&E staining of the tumor and normal tissue cross-sections. The bar represents $200 \mu \mathrm{m}$. The values are expressed as the mean \pm SD, $n=3$ per group.

\section{Antitumor efficacy of FA-IR780-NP in vivo}

The effective phototherapeutic properties of FA-IR780-NP were confirmed earlier in vitro. To confirm the effective phototherapeutic properties of FA-IR780-NP in vivo, SKOV3 tumor-bearing mice were intravenously administered PBS $(200 \mu \mathrm{L})$ as a control, free IR780 $(1 \mathrm{mg} / \mathrm{kg})$, IR780-NP $(30 \mathrm{mg} / \mathrm{kg}$; the corresponding IR780 dose was $1 \mathrm{mg} / \mathrm{kg}$ ) or FA-IR780-NP (30 mg/kg; the corresponding IR780 dose was $1 \mathrm{mg} / \mathrm{kg}$ ). After $3 \mathrm{~d}$ postinjection, the tumor areas were irradiated with a laser $\left(1 \mathrm{~W} / \mathrm{cm}^{2}, 10 \mathrm{~min}\right)$, and temperature changes were monitored and recorded using an infrared thermal imaging camera. As shown in Figure10A and $\mathrm{B}$, due to the superior accumulation, the temperature of the FA-IR780-NP group increased faster and to a higher level than that of the other groups. After confirmation of the phototherapeutic properties of FA-IR780-NP, the phototherapeutic effect was evaluated. The SKOV3 tumor-bearing mice were randomly divided into the following 4 groups ( $\mathrm{n}=6$ per group): control (PBS); FA-IR780-NP (intravenous administration of $30 \mathrm{mg} / \mathrm{kg}$ FA-IR780-NP); NIR laser irradiation(the tumor areas were irradiated with an $808 \mathrm{~nm}$ laser at $1 \mathrm{~W} / \mathrm{cm}^{2}, 10 \mathrm{~min}$ ); and PTT ( $3 \mathrm{~d}$ postinjection of $30 \mathrm{mg} / \mathrm{kg}$ FA-IR780-NP, the tumor areas were irradiated with an $808 \mathrm{~nm}$ laser at $\left.1 \mathrm{~W} / \mathrm{cm}^{2}, 10 \mathrm{~min}\right)$. After the different treatments, the tumor volumes and body weight were monitored.
As indicated in Figure 10C, compared with the control group, the NIR laser irradiation and the FA-IR780-NP administration group showed negligible inhibition of tumor growth. However, the tumors in the PTT group were eradicated in $5 \mathrm{~d}$ after treatment, and no tumor recurrence was observed over $30 \mathrm{~d}$ after treatment (Figure 10D). Due to the excellent tumor-targeting ability of FA-IR780-NP, the surrounding normal tissues avoided serious thermal damage by PTT in the observed period. Specifically, the mice from all four groups showed similar body weight curves over two weeks (Figure 10E). The results indicated that PTT mediated by FAIR780-NP is an effective treatment for ovarian cancer.

Furthermore, the pathological effects of the different intervention modalities at the cellular level were assessed using TUNEL, PCNA, and H\&E staining. As shown in Figure 11A, PCNA-positive cells were dominant in the control, NIR laser irradiation, and FA-IR780-NP groups, which indicated vigorous proliferation, while TUNELpositive cells were dominant in the PTT group, which indicated cell apoptosis. Moreover, a large number of necrotic cells were observed in the PTT group through H\&E staining (Figure 11B). These results indicated that the PTT mediated by FA-IR780-NP killed tumor cells through hyperthermic necrosis and apoptosis.

The modality of PTT has been demonstrated to have the following advantages: 1) tumor selectivity through the 
A

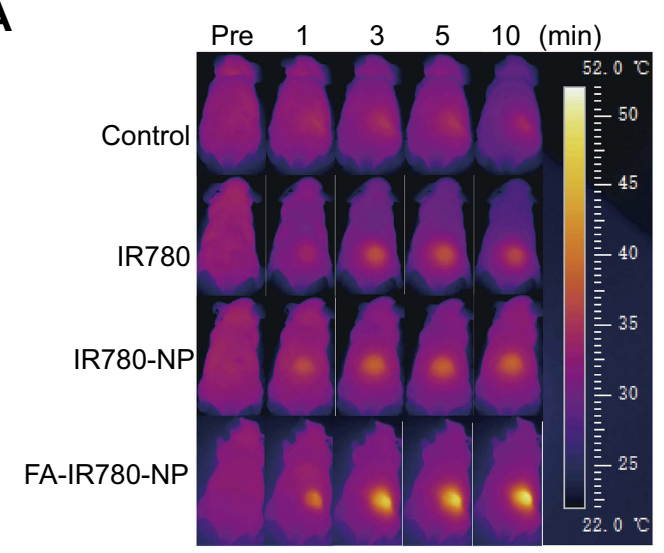

C

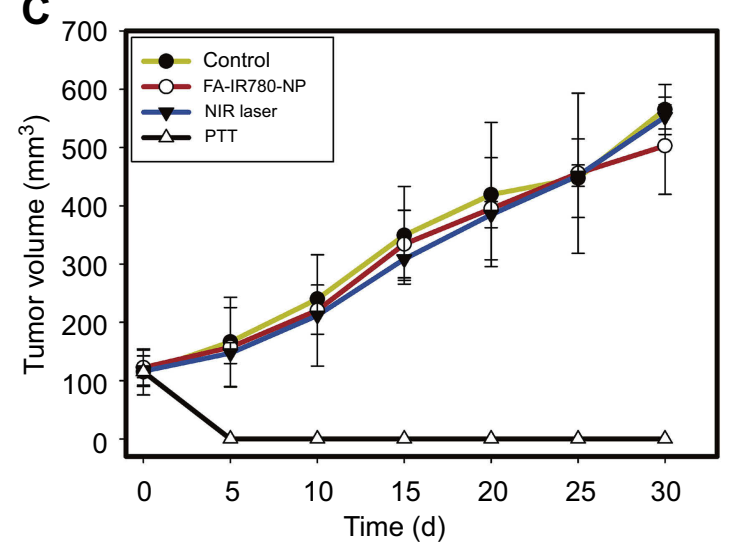

D

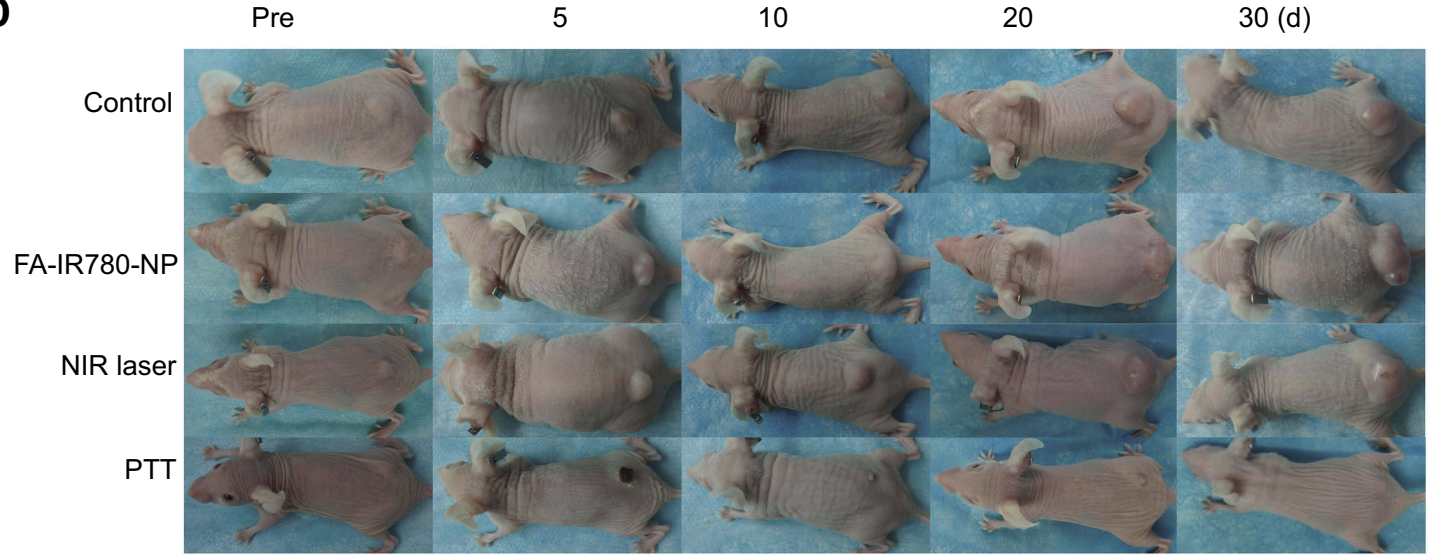

B

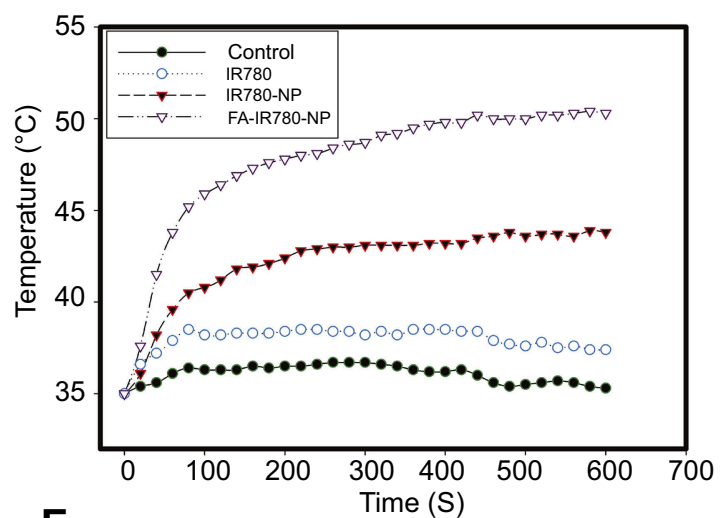

E

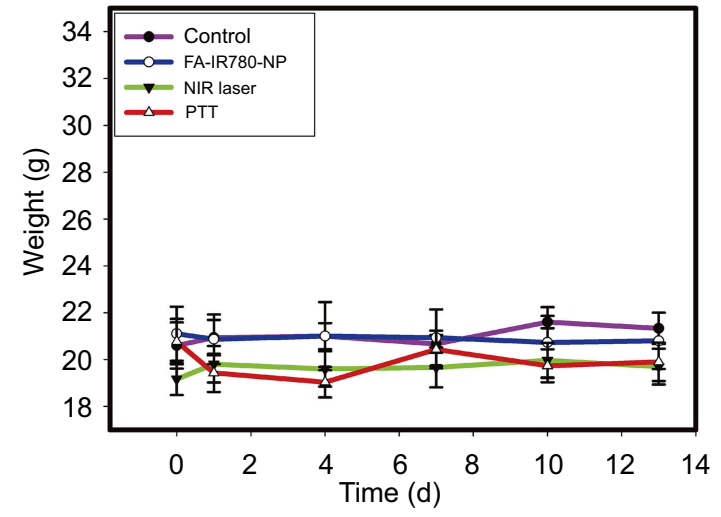

10

Figure 10 Therapeutic effects of PTT mediated by FA-IR780-NP in vivo. (A) Thermal images of SKOV3 tumor-bearing mice from different groups (control, IR780, IR780-NP,

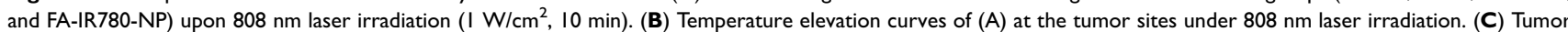
volume curves of the different groups after treatments. (D) Images of tumor-bearing mice in the four groups were captured over a 30 -day period after the different treatments. (E) Body weight curves of the different groups after treatments. The values are expressed as the mean $\pm S D$, $n=6$ per group.

excellent tumor-targeting accumulation of FA-IR780-NP; 2) convenience and safety with NIR light delivery; and 3) real-time monitoring and regulation with a fluorescence stereomicroscope and an infrared thermal imaging camera. Due to these advantages, PTT can potentially be used in ovarian cancer surgery. Surgery can shrink and expose tumors to overcome the penetration depth limitation of PTT, while PTT can eliminate small lesions that cannot be removed by surgery. ${ }^{35,36}$ Multiple small lesions resulting from peritoneal implantation metastasis and adjoining abdomen organs increase the difficulty of eliminating tumors only through surgery. ${ }^{6}$ Even with optimal tumor resection, residual lesions smaller than $1 \mathrm{~cm}$ probably remain. The elimination of these residual lesions through PTT during surgery may potentially improve the outcomes of ovarian cancer patients. 


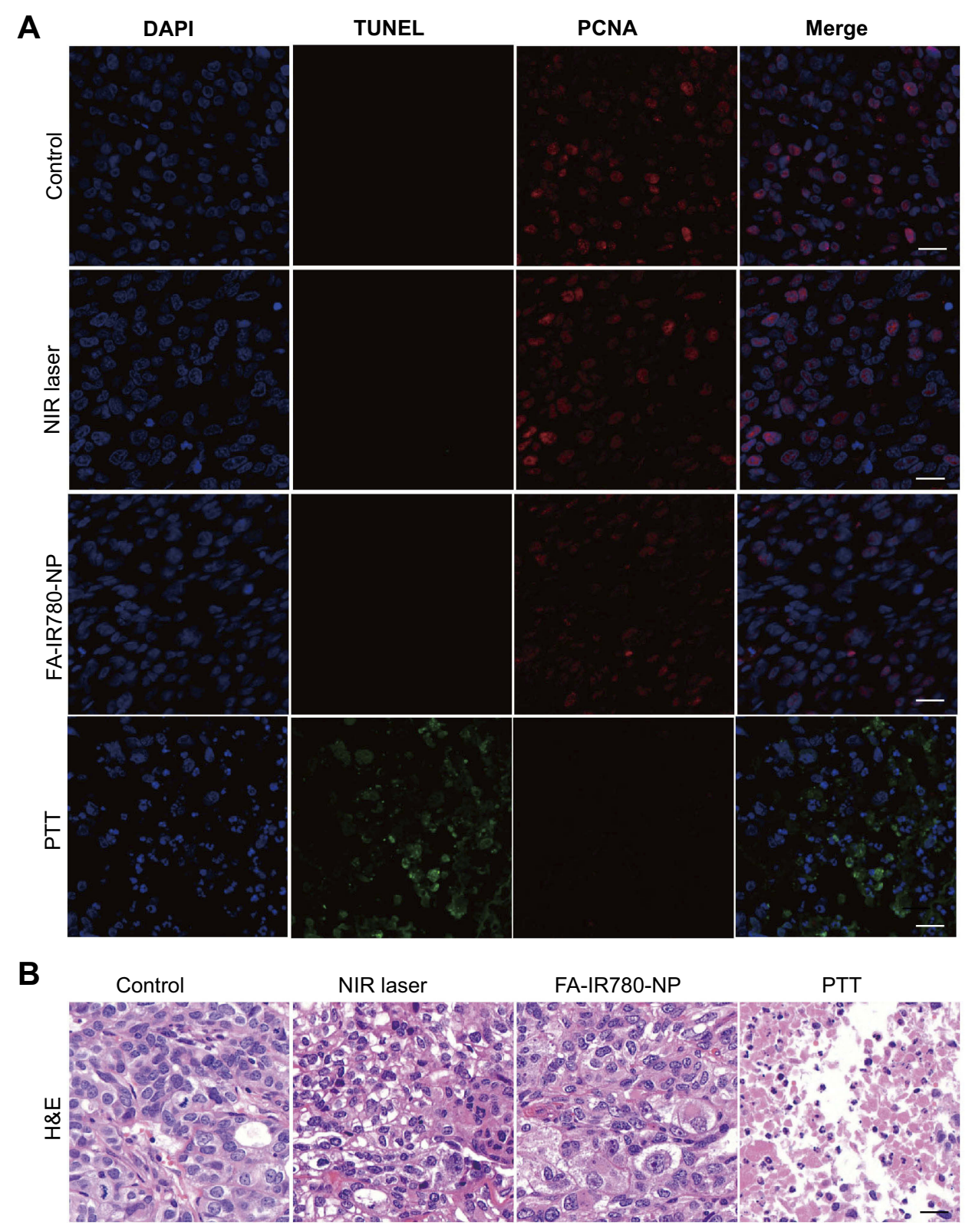

Figure II Pathological effects of the different treatments. (A) PCNA and TUNEL staining of tumor regions after the different treatments. (B) H\&E staining of tumor regions after the different treatments. The white and black bars represent $200 \mu \mathrm{m}, \mathrm{n}=3$ per group.

\section{Conclusions}

We have successfully developed a theranostic FA-IR780NP that provides a new modality for ovarian cancer treatment. On the basis of the EPR effect of NPs, active targeting of FA and selectively accumulation in tumors of IR780, the FA-IR780-NP has excellent tumor-targeting ability. With the aid of tumor targeting and the NIR fluorescence imaging ability, FA-IR780-NP can be used as an indicator for the real-time delineation of small tumor margins $(<1 \mathrm{~cm})$ during surgery. In other words, optimal cytoreductive surgery ( $\leq 1 \mathrm{~cm}$ residuum) could be achieved through the guidance of NIR fluorescence imaging. Furthermore, PTT mediated by FA-IR780-NP effectively eradicated the ovarian cancer tumors that provide a safe adjuvant therapy for residual lesions in surgery that probably remain even with optimal cytoreductive surgery. Otherwise, FA-IR780-NP, with its excellent targeting ability and negligible toxicity in the tested doses, could be used as a new drug delivery system for the diagnosis and treatment of ovarian cancer. Overall, FA-IR780-NP is highly promising for further application in the clinic for ovarian cancer. 


\section{Acknowledgments}

This work was financially supported by the National Natural Science Foundation of China (Grant Nos. 31630026, 81630047, 81771847, 81771845, and 81601513).

\section{Disclosure}

The authors report no conflicts of interest in this work.

\section{References}

1. Chen W, Zheng R, Baade PD, et al. Cancer statistics in China, 2015. Ca-Cancer J Clin. 2016;66(2):115-132. doi:10.3322/caac.21338

2. Siegel RL, Miller KD, Jemal A. Cancer statistics, 2018. Ca-Cancer J Clin. 2018;68(1):7-30. doi:10.3322/caac.21442

3. Bowtell DD, Böhm S, Ahmed AA, et al. Rethinking ovarian cancer II: reducing mortality from high-grade serous ovarian cancer. Nat Rev Cancer. 2015;15(11):668-679. doi:10.1038/nrc4019

4. Chiva LM, Castellanos T, Alonso S, et al. Minimal macroscopic residual disease $(0.1-1 \mathrm{~cm})$. Is it still a surgical goal in advanced ovarian cancer? Int J Gynecol Cancer. 2016;26(5):906-911. doi:10.1097/IGC.0000000000000690

5. Hacker NF, Rao A. Surgery for advanced epithelial ovarian cancer. Best Pract Res Cl Ob. 2017;41:71-87. doi:10.1016/j. bpobgyn.2016.10.007

6. Chang SJ, Bristow RE, Chi DS, Cliby WA. Role of aggressive surgical cytoreduction in advanced ovarian cancer. $J$ Gynecol Oncol. 2015;26(4):336-342. doi:10.3802/jgo.2015.26.4.336

7. Coleman RL, Monk BJ, Sood AK, Herzog TJ. Latest research and treatment of advanced-stage epithelial ovarian cancer. Nat Rev Clin Oncol. 2013;10(4):211-224. doi:10.1038/nrclinonc.2013.5

8. Oronsky B, Ray CM, Spira AI, et al. A brief review of the management of platinum-resistant-platinum-refractory ovarian cancer. Med Oncol. 2017;34(6):103. doi:10.1007/s12032-017-0960-z

9. Cocco E, Shapiro EM, Gasparrini S, et al. Clostridium perfringens enterotoxin C-terminal domain labeled to fluorescent dyes for in vivo visualization of micrometastatic chemotherapy-resistant ovarian cancer. Int J Cancer. 2015;137(11):2618-2629. doi:10.1002/ijc.29632

10. Hoogstins CES, Tummers QRJG, Gaarenstroom KN, et al. A novel tumor-specific agent for intraoperative near-infrared fluorescence imaging: a translational study in healthy volunteers and patients with ovarian cancer. Clin Cancer Res. 2016;22(12):2929-2938. doi:10.1158/1078-0432.CCR-15-2640

11. van Dam GM, Themelis G, Crane LM, et al. Intraoperative tumorspecific fluorescence imaging in ovarian cancer by folate receptoralpha targeting: first in-human results. Nat Med. 2011;17(10):13151319. doi: $10.1038 / \mathrm{nm} .2472$

12. Harmsen S, Teraphongphom N, Tweedle MF, et al. Optical surgical navigation for precision in tumor resections. Mol Imaging Biol. 2017;19(3):357-362. doi:10.1007/s11307-017-1054-1

13. Di Lorenzo G, Ricci G, Severini GM, et al. Imaging and therapy of ovarian cancer: clinical application of nanoparticles and future perspectives. Theranostics. 2018;8(16):4279-4294. doi:10.7150/ thno. 26345

14. Wang H, Li X, Tse BW-C, et al. Indocyanine green-incorporating nanoparticles for cancer theranostics. Theranostics. 2018;8(5):12271242. doi: $10.7150 /$ thno. 22872

15. Veys I, Pop FC, Vankerckhove S, et al. ICG-fluorescence imaging for detection of peritoneal metastases and residual tumoral scars in locally advanced ovarian cancer: a pilot study. J Surg Oncol. 2018;117(2):228-235. doi:10.1002/jso.24807
16. Cheng Z, Al Zaki A, Hui JZ, Muzykantov VR, Tsourkas A. Multifunctional nanoparticles: cost versus benefit of adding targeting and imaging capabilities. Science. 2012;338(6109):903-910. doi:10.1126/science. 1226338

17. Pelaz B, Alexiou C, Alvarez-Puebla RA, et al. Diverse applications of nanomedicine. ACS Nano. 2017;11(3):2313-2381. doi:10.1021/ acsnano.6b06040

18. Kunjachan S, Pola R, Gremse F, et al. Passive versus active tumor targeting using RGD- and NGR-modified polymeric nanomedicines. Nano Lett. 2014;14(2):972-981. doi:10.1021/n1404391r

19. Luo X, Li B, Zhang X, et al. Dual-functional lipid-like nanoparticles for delivery of mRNA and MRI contrast agents. Nanoscale. 2017;9 (4):1575-1579. doi:10.1039/c6nr08496f

20. O'Shannessy DJ, Somers EB, Smale R, et al. Expression of folate receptor- $\alpha$ (FRA) in gynecologic malignancies and its relationship to the tumor type. Int $J$ Gynecol Pathol. 2013;32(3):258-268. doi:10.1097/PGP.0b013e3182774562

21. Thomas RG, Jeong YY. NIRF heptamethine cyanine dye nanocomplexes for multi modal theranosis of tumors. Chonnam Med J. 2017;53(2):83-94. doi:10.4068/cmj.2017.53.2.83

22. Yan F, Duan W, Li Y, et al. NIR-laser-controlled drug release from DOX/IR-780-loaded temperature-sensitive-siposomes for chemophotothermal synergistic tumor therapy. Theranostics. 2016;6 (13):2337-2351. doi:10.7150/thno.14937

23. Wang S, Ma X, Hong X, et al. Adjuvant photothermal therapy inhibits local recurrences after breast-conserving surgery with little skin damage. ACS Nano. 2018;12(1):662-670. doi:10.1021/acsnano.7b07757

24. Yang X, Li H, Qian C, et al. Near-infrared light-activated IR780-loaded liposomes for anti-tumor angiogenesis and photothermal therapy. Nanomedicine. 2018;14(7):2283-2294. doi:10.1016/j.nano.2018.06.011

25. Guo F, Yu M, Wang J, et al. Smart IR780 theranostic nanocarrier for tumor-specific therapy: hyperthermia-mediated bubble-generating and folate-targeted liposomes. ACS Appl Mater Inter. 2015;7 (37):20556-20567. doi:10.1021/acsami.5b06552

26. Li S, Johnson J, Peck A, et al. Near infrared fluorescent imaging of brain tumor with IR780 dye incorporated phospholipid nanoparticles. J Transl Med. 2017;15(1):18. doi:10.1186/s12967-016$1115-2$

27. Yun SH, Kwok SJJ. Light in diagnosis, therapy and surgery. Nat Bio Eng. 2017;1:0008. doi:10.1038/s41551-016-0008

28. Li H, Wang K, Yang X, et al. Dual-function nanostructured lipid carriers to deliver IR780 for breast cancer treatment: anti-metastatic and photothermal anti-tumor therapy. Acta Biomater. 2017;53:399413. doi:10.1016/j.actbio.2017.01.070

29. Li M, Teh C, Ang CY, et al. Near-infrared light-absorptive stealth liposomes for localized photothermal ablation of tumors combined with chemotherapy. Adv Funct Mater. 2015;25(35):5602-5610. doi:10.1002/adfm.201502469

30. Li H, Yang X, Zhou Z, et al. Near-infrared light-triggered drug release from a multiple lipid carrier complex using an all-in-one strategy. J Control Release. 2017;261:126-137. doi:10.1016/j. jconrel.2017.06.029

31. Zhang C, Wang S, Xiao J, et al. Sentinel lymph node mapping by a near-infrared fluorescent heptamethine dye. Biomaterials. 2010;31 (7):1911-1917. doi:10.1016/j.biomaterials.2009.11.061

32. Li X, Schumann C, Albarqi HA, et al. A tumor-activatable theranostic nanomedicine platform for NIR fluorescence-guided surgery and combinatorial phototherapy. Theranostics. 2018;8(3):767-784. doi:10.7150/ thno. 21209

33. Wong AW, Fite BZ, Liu Y, et al. Ultrasound ablation enhances drug accumulation and survival in mammary carcinoma models. $J$ Clin Invest. 2016;126(1):99-111. doi:10.1172/JCI83312

34. Suk JS, Xu Q, Kim N, et al. PEGylation as a strategy for improving nanoparticle-based drug and gene delivery. Adv Drug Delivery Rev. 2016;99(Pt A):28-51. doi:10.1016/j.addr.2015.09.012 
35. Zhu H, Cheng P, Chen P, et al. Recent progress in the development of near-infrared organic photothermal and photodynamic nanotherapeutics. Biomater Sci. 2018;6(4):746-765. doi:10.1039 c7bm01210a
36. Qi J, Chen C, Zhang X, et al. Light-driven transformable optical agent with adaptive functions for boosting cancer surgery outcomes. Nat Commun. 2018;9(1):1848. doi:10.1038/s41467-01804222-8 


\section{Supplementary material}

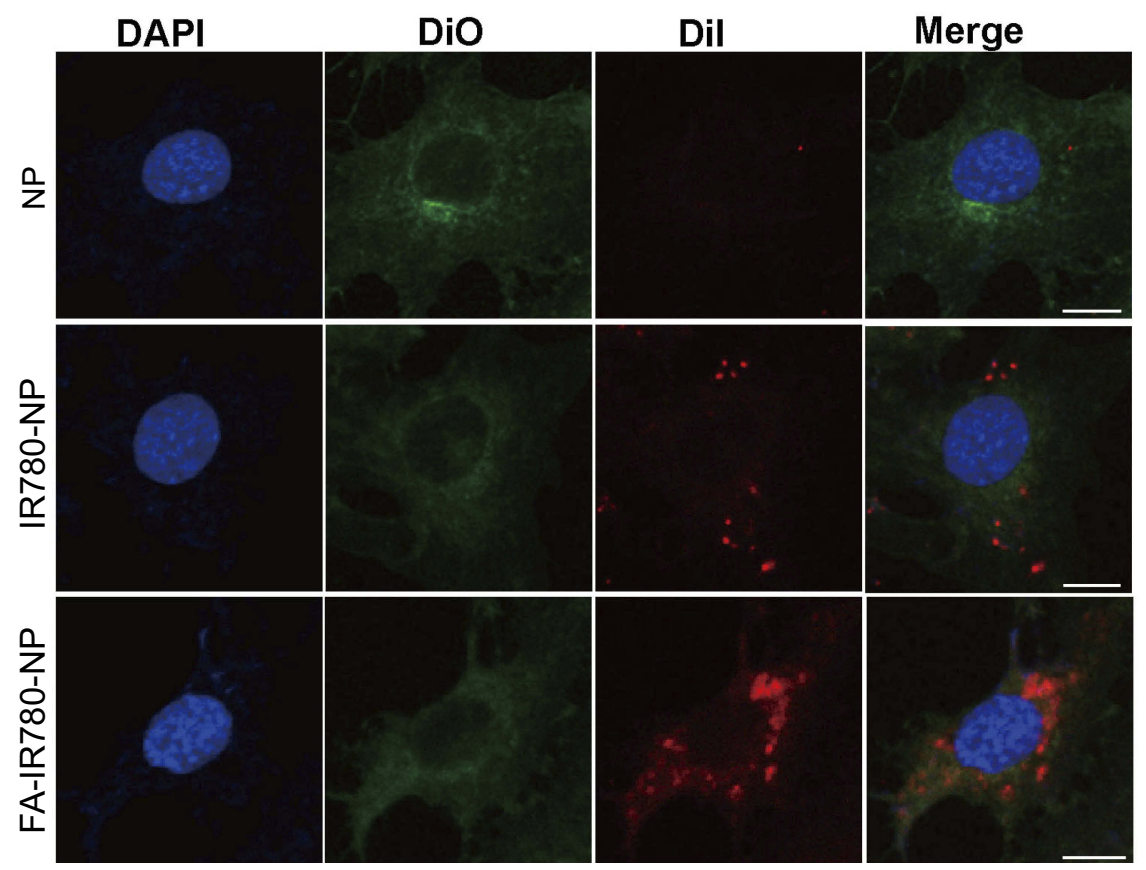

Figure SI Fluorescence images of a single SKOV3 cell incubated with NPs (NP, IR780-NP, FA-IR780-NP). Blue (DAPI) represents cell nuclei; green (Dio) reveals the cytomembrane; whereas the red (Dil) dots imply Dil-labeled NPs. The scale bars represent $5 \mu \mathrm{m}$.

\section{Publish your work in this journal}

The International Journal of Nanomedicine is an international, peerreviewed journal focusing on the application of nanotechnology in diagnostics, therapeutics, and drug delivery systems throughout the biomedical field. This journal is indexed on PubMed Central, MedLine, CAS, SciSearch ${ }^{\circledR}$, Current Contents ${ }^{\circledR} /$ Clinical Medicine,
Journal Citation Reports/Science Edition, EMBase, Scopus and the Elsevier Bibliographic databases. The manuscript management system is completely online and includes a very quick and fair peer-review system, which is all easy to use. Visit http://www.dovepress.com/ testimonials.php to read real quotes from published authors. 